\title{
Rôle de la kinésithérapie maxillo-faciale dans la prise en charge multidisciplinaire des cancers des voies aéro - digestives supérieures
}

\author{
Varaud $\mathrm{N}^{1}$, Léon $\mathrm{H}^{2}$, Delarbre $\mathrm{B}^{2}$, Triay $\mathrm{JA}^{2}$, Lozza $\mathrm{J}^{2}$ \\ ${ }^{1}$ Masseur kinésithérapeute spécialisée dans le domaine maxillo-faciale, Nîmes, France \\ ${ }^{2}$ Service d'Odontologie et de Prothèse maxillo-faciale, CHU, Nîmes, France \\ n.varaud@yahoo.fr
}

L'odontologiste participe à la prise en charge des cancers des voies aéro-digestives supérieures. Il intervient dans la préparation des traitements anti-cancéreux mais aussi dans la prévention et le suivi des complications de ces mêmes traitements. Les complications peuvent être très invalidantes. La kinésithérapie maxillo-faciale constitue une réponse efficace à certaines d'entre elles et augmente considérablement la qualité de vie du patient. L'expérience montre que peu de patients bénéficient de ces soins après un traitement anti-cancéreux. Il est donc important que l'odontologiste connaisse cette discipline et prescrive une prise en charge kinésithérapique adaptée à ses patients.

Nous vous présentons plusieurs cas cliniques illustrant les possibilités thérapeutiques de la kinésithérapie maxillo-faciale carcinologique. 